\title{
Characterization of the Specific Absorption Rate of the Cell Phone in the Human Head, Using the LN-FDTD Method
}

\author{
Adelson Bezerra de Medeiros ${ }^{\mathrm{a}}$, Jandecy Cabral Leite ${ }^{\mathrm{b}}$ \\ ${ }^{a}$ Dr., Universidade Federal do Pará (UFPA). (medeiros@ufpa.br) \\ ${ }^{b}$ Dr., Instituto de Tecnologia e Educação Galileo da Amazônia (ITEGAM). Brasil. (jandecy.cabral@itegam.org.br).
}

\begin{abstract}
In this work we show how the study of the absorption of electromagnetic energy will be approached in biological tissues of the human head, originating from cell phones in a $900 \mathrm{MHz}$ band, and the development of a program to calculate the Specific Absorption Rate (SAR) in the cell phone user's head. The implementation of the Local Non-Orthogonal Finite-Difference Time Domain method is presented (LN-FDTD), and the use of Uniaxial Perfectly-Matched Layer (UPML), comparing the results obtained with the existing results in the literature.
\end{abstract}

Keywords: Cell phone, Biological Effects, Specific Absorption Rate (SAR), Local Non-Orthogonal Finite-Difference Time Domain (LN-FDTD), Uniaxial Perfectly-Matched Layer (UPML), Non-ionizing radiation.

\section{INTRODUCTION}

The popularity of the mobile telephony in the globalized world has caused a growing demand of the people's needs in the globalized society in trying to reduce the distances among them, what made the mobile communication easier and at the same time provided new life perspectives. Even with all the progress on the mobile telephony, through their benefits and applications on behalf of the society, this kind of system causes damages to the human health, since, in several situations, these gadgets can emit high potencies when they are very close to the user's head. In these cases, due to the great exposition to the electromagnetic radiations which people are submitted, a considerable portion of the irradiated energy is absorbed by the head tissues: skin, fat, bone and brain etc, some of which are formed by extremely sensitive elements which are very important for the human being, as for instance: the neurons, chromosomes etc. This absorption of non-ionizing electromagnetic energy can provoke basically two effects: (a) The "Thermal Effects" and (b) "Non-Thermal Effects" [1-2].

The thermal effects have been known for at least 50 years (fifty years). They originate from the heating of the tissue. The radiation is not only absorbed at the level of the skin, but also in deeper levels of the body, causing a temperature increase which is not noticed by the natural thermal sensors because it's superficially located. That generated internally heat, depending on the amount of exposition time, the intensity of the field and the thickness of the tissue, cannot be compensated and it causes serious damages: (a) increase of the temperature in the organs, (b) alterations in their electric operation due to modifications in the chemical operation of the cells and (c) alterations in the mechanism of electric signs transmission, from the brain to the muscles, according to Salles [3].

The exhibition limits vary according to the frequency, partly for considering the resonance and also for considering the smallest depth of penetration of the electromagnetic fields in higher frequencies. The resonance is important because when this phenomenon happens, it results in larger absorption of energy, for instance: an adult's head can present resonance in bands from 400 to $500 \mathrm{MHz}$, and in a child's head the resonance can happen in bands from 700 to $800 \mathrm{MHz}$ (ICNIRP, 1998). The non-thermal effects are biochemical or electro-physical effects caused directly by the induced electromagnetic fields. These effects are still part of studies which present conflicting results in the international scientific literature. The effects that were clearly demonstrated include the alteration in the ions flow through the membranes of the cells (affecting particularly the electrophysiologic properties of the nervous cells) alterations in the synthesis of DNA and in the transcription of RNA and effects in the normal cells responses to the signaling molecule (including hormones, neurotransmitters and growth factors). Alterations in 
the flow of calcium in the cells, in the blood-brain barrier that protects the brain from certain toxins and in the development of brain tumors which were reported by (Fischetti, 1993).

In the problem we are proposing, the non-ionizing radiation is used to determine the Specific Absorption Rate of the cell phone in the human head, varying the distance from the cell phone to the head and the frequency of the cell phone. We used the Local Non-Orthogonal Finite-Difference Time Domain method (LN-FDTD) [4], which contributed to the development of an algorithm to obtain the (SAR) of the cell phone in the user's head.

The main contribution of this work is to present a numerical method to solve the staircase problem in an ellipsoid surface and the obtaining of the results that are more precise and time saving in the computational field.

\section{THEORETICAL IMPLEMENTATION OF THE LN- FDTD METHOD}

We defined an irregular non-orthogonal structured grid composed of meshes denominated primary and secondary. The primary mesh is projected to adapt the geometry of the problem, the one that can be accomplished by the use of a generator of adaptive meshes. This mesh can also be chosen to align with the components of the electric or magnetic field, depending on the contour/outline condition that is imposed in the physical geometry. Then, the second mesh is generated by the connection of the baricenters of the primary mesh cells.

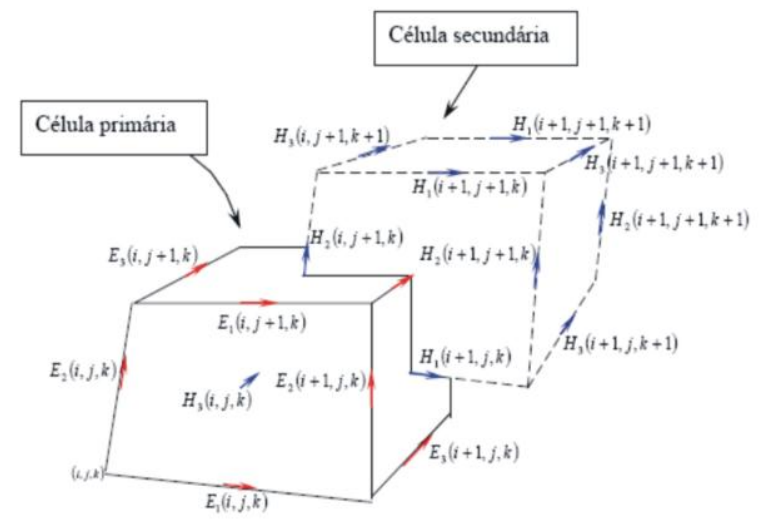

Fig. 1. Primary and secondary cell of an irregular non-orthogonal structured mesh.

For the geometries that can be discretized by this kind of grid, it is defined the unitary vectors of base, $A_{i}(1,2,3)$, for the edges of the primary and secondary meshes cells, according to figure 2 .

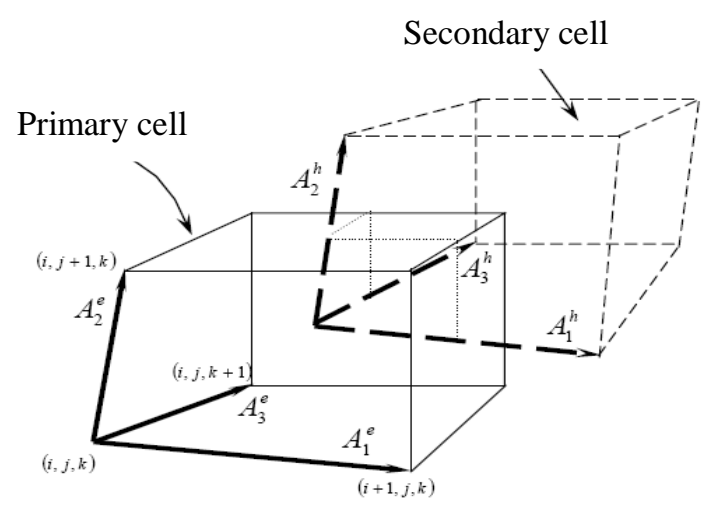

Fig. 2. Definition of the unitary vectors of base for the primary and secondary cells in the system of local curvilinear coordinates.

Because the equations of Maxwell in the integral form are applied naturally in a non-orthogonal irregular space they will be used to obtain the components for updating the electric and magnetic fields in the time domain, using the LN-FDTD method. Being considered an isotropic environment and free from sources [5], it can be written as:

$$
\begin{aligned}
& -\frac{\partial}{\partial_{t}} \int_{\Omega} \mu H \cdot d a=\oint_{\partial \Omega} \mathrm{E} \cdot \mathrm{dl} \\
& \frac{\partial}{\partial_{t}} \int_{\Omega} \epsilon E \cdot d a=\oint_{\partial \Omega} \mathrm{H} \cdot \mathrm{dl}
\end{aligned}
$$

The electric field $\mathrm{E}(i, j, k)$ in a point $(i, j, k)$ of the grid which can be expressed in terms of the two bases, respectively, as,

$$
\begin{gathered}
\mathrm{E}(i, j, k)=\sum_{j=1}^{3} \mathrm{E}^{j}(i, j, k) \mathrm{A}_{j}(i, j, k) \\
\mathrm{E}(i, j, k)=\sum_{j=1}^{3} \mathrm{E}_{j}(i, j, k) \mathrm{A}^{j}(i, j, k)
\end{gathered}
$$

In (3) and (4), the coefficients $\mathrm{E}^{i}$ and $\mathrm{E}_{i}$ are called contravarying and co-varying components, respectively, of the electric 
field. The co-varying component $\mathrm{E}_{i}(i, j, k)$ represents the flow of $\mathrm{E}(i, j, k)$ along the edge $i$, and $\mathrm{E}^{i}(i, j, k)$ represents the total flow of the electric field passing through the face $i$.

Starting from the physical interpretation, we can write the expressions for the fields $(\mathrm{E} e \mathrm{H})$, that appear in the line integral (right side) of the equations (1) and (2). We solve equation (1) using the LN-FDTD method, for the contra-varying component of the magnetic field $\mathrm{H}^{i}(i, j, k)^{n+\frac{1}{2}}$, assuming that it is constant on the face of each cell, and written in function of the volume of the cell, resulting in,

$$
\begin{aligned}
\mu \frac{\partial}{\partial t} \mathrm{H}^{1}(i, j, k)^{n} V & =\left(\mathrm{E}_{3}(i, j+1, k)^{n}-\mathrm{E}_{3}(i, j, k)^{n}\right) \\
& -\left(\mathrm{E}_{2}(i, j, k+1)^{n}-\mathrm{E}_{2}(i, j, k)^{n}\right)
\end{aligned}
$$

In the system of local curvilinear coordinates, the volume $V$ is defined for each contra-varying component, starting from the vectors of unitary base which define the cell in the picture (2).

For the calculation of the relative volume of a certain face, it is necessary to know the unitary base vector of the normal contra-varying component to this face and the four vectors of unitary base, of the dual mesh, which define the face through which the vectorial contra-varying field passes. This way, the volume for the component $\mathrm{H}^{1}$, in agreement with the pictures (1) and (2) is given by:

$V_{1}^{h}=\mathrm{A}_{1}^{h}(i, j, k)\left[\left\{\begin{array}{l}\left(\frac{\mathrm{A}_{2}^{e}(i, j, k+1)+\mathrm{A}_{2}^{e}(i, j, k)}{2}\right) \\ \times\left(\frac{\mathrm{A}_{3}^{e}(i, j+1, k)+\mathrm{A}_{3}^{e}(i, j, k)}{2}\right)\end{array}\right\}\right.$

We can obtain the updating expression for the contravarying component of the magnetic field $\mathrm{H}^{1}(i, j, k)^{n+\frac{1}{2}}$, which is done by replacing (6) in (5) resulting in,

$$
\begin{aligned}
\mathrm{H}^{1}(i, j, k)^{n+\frac{1}{2}} & =\mathrm{H}^{1}(i, j, k)^{n-\frac{1}{2}}-\frac{\Delta t}{\mu V_{1}^{h}(i, j, k)} \\
& \left\{\left[\mathrm{E}_{3}(i, j+1, k)^{n}-\mathrm{E}_{3}(i, j, k)^{n}\right]\right. \\
& \left.-\left[\mathrm{E}_{2}(i, j, k+1)^{n}-\mathrm{E}_{2}(i, j, k)^{n}\right]\right\}
\end{aligned}
$$

The other contra-varying component of the magnetic field $\mathrm{H}^{2}(i, j, k)^{n+\frac{1}{2}}$ and $\mathrm{H}^{3}(i, j, k)^{n+\frac{1}{2}}$, and the vectors $V_{2}^{h}$ and $V_{3}^{h}$ are obtained in a similar way. The solution of the equation (2), by the LN- FDTD method, to calculate the contravarying component of the electric field $\mathrm{E}^{1}(i, j, k)^{n+1}$

$$
\begin{array}{r}
\mathrm{E}^{1}(i, j, k)^{n+1}=\mathrm{E}^{1}(i, j, k)^{n}+\frac{\Delta t}{\varepsilon(i, j, k) V_{1}^{e}(i, j, k)} \square \\
\left\{\begin{array}{l}
{\left[\mathrm{H}_{3}(i, j, k)^{n+\frac{1}{2}}-\mathrm{H}_{3}(i j-1, k)^{n+\frac{1}{2}}\right]-} \\
{\left[\begin{array}{l}
\mathrm{H}_{2}(i, j, k)^{n+\frac{1}{2}}-\mathrm{H}_{2}(i, j, k-1)^{n+\frac{1}{2}}
\end{array}\right]}
\end{array}\right\}
\end{array}
$$

The contra-varying components of the electric field $\mathrm{E}^{2}(i, j, k)^{n+\frac{1}{2}}$ and $\mathrm{E}^{3}(i, j, k)^{n+\frac{1}{2}}$ are obtained in a similar way, with their respective volumes given by:

$$
V_{1}^{e}(i, j, k)=\mathrm{A}_{1}^{e}(i, j, k)\left[\left\{\begin{array}{l}
{\left[\frac{\mathrm{A}_{2}^{h}(i, j, k)+\mathrm{A}_{2}^{h}(i, j, k-1)}{2}\right]} \\
\times\left[\frac{\mathrm{A}_{3}^{h}(i, j, k)+\mathrm{A}_{3}^{h}(i, j-1, k)}{2}\right]
\end{array}\right\}\right.
$$

The volumes $V_{2}^{e}$ and $V_{3}^{e}$ are obtained in a similar way. It is necessary to convert the contra-varying components of the electric field into co-varying components, so that the contravarying components of the field $\mathrm{H}$ can make calculations, and vice-versa.

\section{DESCRIPTION OF THE MODEL}

The development of this work will happen inside an UPML [6], whose walls are covered by absorbent layers (ABC), which plays the role of the contour/outline conditions. The interior of the UPML is called analysis area, and a human head 
Adelson Bezerra de Medeiros \& Jandecy Cabral Leite / ITEGAM-JETIA Vol.01, Nº 01, pp.61-66. Março, 2015.

will be put with its respective dielectric properties: conductivity, permeability and permissiveness of each tissue, distant $9 \mathrm{~mm}$, $18 \mathrm{~mm}$ and $27 \mathrm{~mm}$ from the dipole of variable frequency of
$900 \mathrm{MHz}$ and $1800 \mathrm{MHz}$, which will measure the electric field on each point of the cell, in agreement with Figure 3.

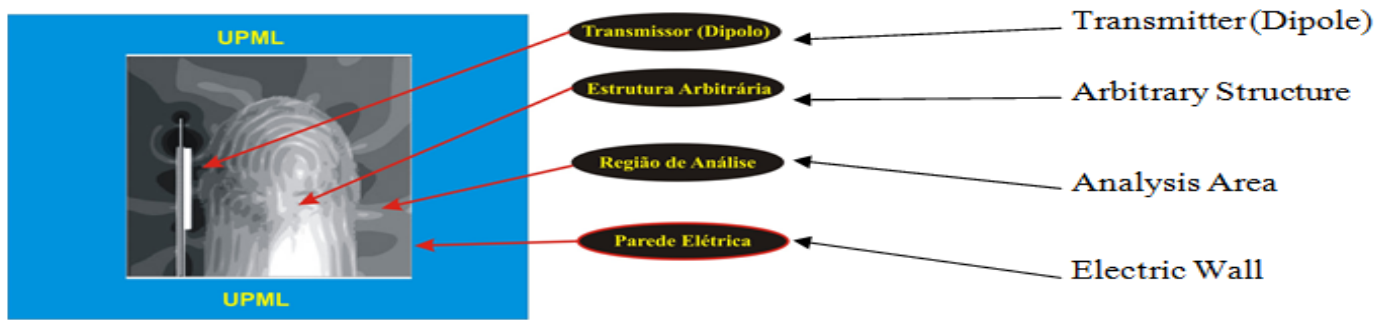

Fig 3. UPML model for the implementation of the LN-FDTD technique.

\section{DESCRIPTION OF THE LN-FDTD MODEL OF THE HUMAN HEAD.}

In this work we make a description of the data and the development of the LN-FDTD numerical model, using the human head experiment. The model is detailed with the different types of tissues: skin, fat, leather-hairy, bone, blood, etc., and with their respective dielectric properties. The human head model validated in the literature with the following dimensions: 264 height $\mathrm{mm}, 177$ width $\mathrm{mm}$ and 240 depth $\mathrm{mm}$. They were obtained from available medical images. A total of 226 images of axial cuts were used, with spacing of $9 \mathrm{~mm}$, yielded by the "Ofir Loyola Hospital", and recommended by the research laboratory AT Telstra Research Laboratories (TLR) [7] each image is composed of $177 \mathrm{X} 240$ points or pixels, composing a mesh of 42.480 cells. For a better aid in the discretization of the tissues, images of computerized tomography and magnetic resonance were used. It has been observed that the pick of SAR is in the base of the nose. This pick also coincides with the presence of the highest tissues of the conductivity: skin, blood, and cartilage, in agreement with the values of the table 1 [7]. Table I. Delectric properties of the human head tissues

\begin{tabular}{|l|c|c|c|}
\hline Tissue & $\begin{array}{c}\text { Conductivity } \\
\left(\Omega^{-1} \mathrm{~m}^{-1}\right)\end{array}$ & $\begin{array}{c}\text { Relative } \\
\text { Permissiveness }\end{array}$ & $\begin{array}{c}\text { Mass Density } \\
\left(\mathrm{kg} / \mathrm{m}^{3}\right)\end{array}$ \\
\hline Cartilage & 0.782 & 42.65 & 1000 \\
\hline Muscle & 0.969 & 55.95 & 1050 \\
\hline Eye & 1.900 & 70.00 & 1000 \\
\hline Brain \& Nerve & 0.766 & 45.80 & 1050 \\
\hline Skin & 0.867 & 41.40 & 1000 \\
\hline Blood & 1.180 & 62.00 & 1000 \\
\hline Skull & 0.242 & 16.62 & 1850 \\
\hline
\end{tabular}

\section{RESULT OF THE HUMAN HEAD MODEL.}

The effects of the non-ionizing electromagnetic radiation in the human head are being studied; as the distribution of electric field and SAR. The standard user's head modeling with the following dimensions: $264 \mathrm{~mm}$ of height (from the chin to the head's top), $177 \mathrm{~mm}$ of width (from ear to ear), and 240 depth $\mathrm{mm}$ (from nose to the rear part of the head) which was constituted by 89 plane sections.

They were obtained from available medical images. A total of 226 images of axial cuts were used, with spacing of 9 mm, yielded by the "Ofir Loyola Hospital."

Each image is composed of 177 x 240 points or pixels, composing a mesh of 42480 cells. For a better aid in the discretization of the tissues, images of computerized Tomography and Magnetic Resonance were used. Initially, we made simulations for the problem consisting of a uniformly plane wave interacting with the human head. We adopted, for an experimental simulation, a non-homogeneous environment, with the dielectric constants of the table 1 , and, for the frequencies of $900 \mathrm{MHz}$ and with a potency of $1 \mathrm{~W}, 0.6 \mathrm{~W} 0.25 \mathrm{~W}$, that were recommended by IEEESCC-34/S-2. For a simple model of human head in 2-D with an axial cut in the plan xy, excited by a dipole antenna $90 \mathrm{~mm}$ long.

The variation of the distance, from the antenna to the head; $9 \mathrm{~mm}$ guided along the axis $\mathrm{z}$. The acquired results regarding the simulation of the electric field and SAR, after 1750 interactions, in the frequencies of $900 \mathrm{MHz}$. For a potency of $1 \mathrm{~W}$, $0.6 \mathrm{~W}, 0.25 \mathrm{~W}$ and in the frequency of $900 \mathrm{MHz}$, in agreement with the table 2 .

Table 2. Frequency of operation.

\begin{tabular}{|c|c|c|c|c|}
\hline $\begin{array}{c}\text { Frequency } \\
\text { of } \\
\text { operation }\end{array}$ & $\begin{array}{c}\text { Distance } \\
(\mathrm{mm})\end{array}$ & $\begin{array}{c}\text { Potency } \\
\text { Antenna } \\
\text { Exit } \\
(\mathrm{W})\end{array}$ & $\begin{array}{c}\text { Electric } \\
\text { Field } \\
\text { Medium } \\
(\mathrm{V} / \mathrm{m})\end{array}$ & $\begin{array}{c}\text { SAR } \\
\text { Medium } \\
(\mathrm{W} / \mathrm{Kg})\end{array}$ \\
\hline \multirow{3}{*}{ 900MHZ } & \multirow{2}{*}{9} & 1.0 & 126 & 15.4 \\
\cline { 3 - 5 } & & 0.6 & 75.6 & 5.56 \\
\cline { 2 - 5 } & 0.25 & 31.5 & 0.96 \\
\hline
\end{tabular}

Based on the values of the table 2, the graphic SAR versus distance are built for the bands of frequency of $900 \mathrm{MHz}$ which were chosen in the column form, and SAR versus 1750 interactions, in which the graphic of the pictures (4) and (5) were 
Adelson Bezerra de Medeiros \& Jandecy Cabral Leite / ITEGAM-JETIA Vol.01, Nº 01, pp.61-66. Março, 2015.

chosen, to give a better understanding of the acquired data during the simulation. Considering the picture (4) for the band of 900 $\mathrm{MHz}$, we can observe that the acquired results in relation with the potencies of $1 \mathrm{~W}$ and $0.6 \mathrm{~W}$ are above the expected; while the data relative to the potency of $0.25 \mathrm{~W}$ in the table (2) show the values of SAR within the limit established by the international agencies and by the national agency ANATEL. In the potencies of $1 \mathrm{~W}$ and $0.6 \mathrm{~W}$ versus the distances of $9 \mathrm{~mm}$, SAR is above the norms of the international organizations like ICNIRP [8] and also of the norms of the national agency ANATEL [9]; while, for the potencies of $0.25 \mathrm{~W}$, in the distance of $9 \mathrm{~mm}$, it's within the maximum limit allowed by law. Most of the results were superior to the limit established by ICNIRP of $2 \mathrm{~W} / \mathrm{Kg}$ for the population in general.

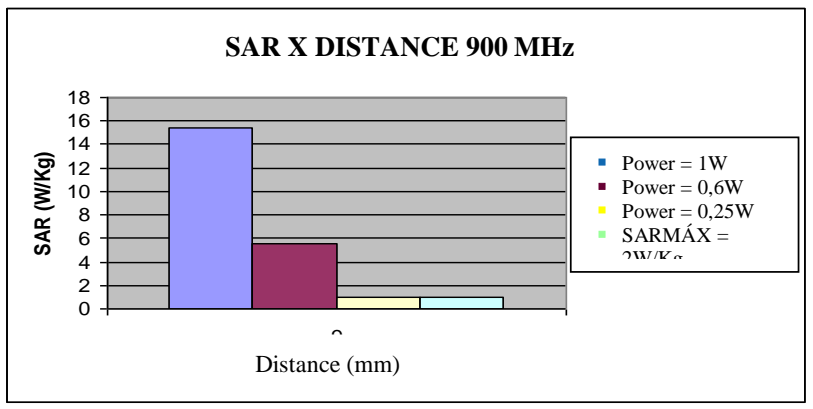

Fig.4. Graphic of the SAR Columns versus distance for a band of $900 \mathrm{MHz}$

We analyzed that some areas of the brain present larger or smaller absorption, as displayed on (Figure 5).

\section{CONCLUSION}

In this work the development of a numerical LN-FDTD method is described (Local Non-Orthogonal Finite-Difference Time Domain (LN-FDTD)), that it is used to determine the absorption of electromagnetic energy in the human head's area. We also supply a study concerning the main aspects of the algorithm to quantify the specific absorption rate (SAR) in the human head.

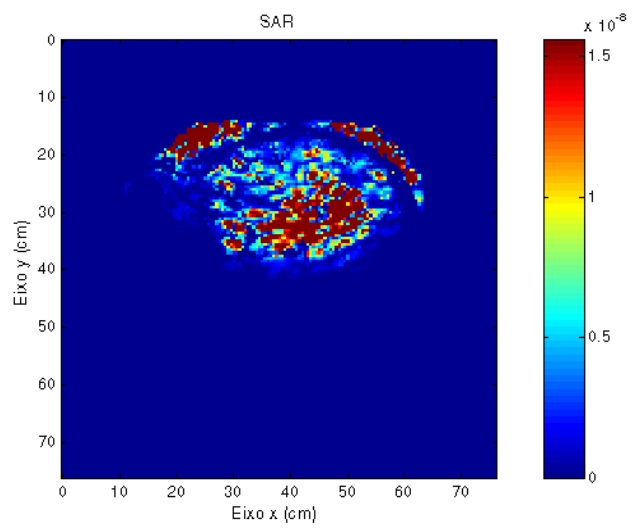

Fig. 5. Present larger or smaller absorption
The results prove the usefulness of the method in the capacity to verify the precision and the efficiency in the solution of problems that involve non-homogeneous materials.

They also indicated a significant influence of several types of tissues that form the human head, and important factors when interpreting the experimental results, particularly (for a plane wave, whose frequency is $900 \mathrm{MHz}$ ), for a medium rate of the head's axial plan.

The values of SAR pick are significantly higher for the areas; eyes and nose of the heterogeneous human head's model.

The results presented for SAR, in the potency of $1 \mathrm{~W}$ and $0.6 \mathrm{~W}$ are above the values established for safety's norms; while the value of $0.25 \mathrm{~W}$ satisfies the demands of the international agencies such as; ICNIRP and ANATEL.

\section{REFERENCES}

[1] Salles, A.A. "Riscos á saúde provocados pelos telefones celulares". EGATEA, Revista da Escola de Engenharia UFRGS, vol. 24, no 1, pp.7-16 (1997).

[2] Salles, A.A.A, Fernandez, C. Bonadiman, M. (2002) “ Simulações do Campo Distante e da SAR na Cabeça do Usuário do Telefone Celular para Antenas Convencionais e Planares". In: E. Fontana e A.J.B de Oliveira. [ed]. Anais do X Simpósio Brasileiro de Microondas e Optoeletronica SBMO2002, Recife, vol.1.p.347-351.

[3] A. A. Salles, C. Fernández, and M. Bonadiman, (2003) "Simulações da SAR na cabeça e antena planares para telefones móveis," Revista Brasileira de Engenharia Biomédica, vol. 19, nº 2, pp. 77-90.

[4] R. Holland, "Finite-Difference solution of Maxwell's equations in generalized nonorthogonal coordinates," IEEE Trans. Nucl. Sci, vol. 6, Dec. 1983.

[5] J.F.Lee Modeling Three-Dimensional Discontinuities in waveguides Using Non-orthogonal FDTD Algorithm. IEEE Trans. On Microwave Theory and Techniques, vol. MTT-40, n², Feb,(1992).

[6] S. D. Gedney, "An Anisotropic Perfectely Matched LayerAbsorbing Medium for the Truncation of FDTD Lattices," IEEE Trans. Antennas Propagat., pp. 1630-1639, Dec. 1996.

[7] R. L Mclntosh, R. McKenzie, and A. Carratelli, "The Numerica Evaluation of a SAR Measurement Phantom at 
the Telstra Research Laboratories". WARS02 - Workshop on the Applications of Radio Science.[Online]. Available: http://www.ips.gov.au/IPSHosted/NCRS/wars /wars2002/proceedings/index.

[8] International Commission on Non-ionizing Radiation Protection (ICNIRP). "Guidelines for Limiting Exposure to Time-Varying Electric, Magnetic, and Electromagnetic Fields (up to 300GHz)", Health Physics, Vol.77, No. 4., Apr. 1998, pp. 494-522. 